\title{
Antidepressants and Hepatotoxicity: A Cohort Study among 5 Million Individuals Registered in the French National Health Insurance Database
}

\author{
Sophie Billioti de Gage ${ }^{1,2}\left({ }^{1} \cdot\right.$ Cédric Collin $^{1} \cdot$ Thien Le-Tri $^{1} \cdot$ Antoine Pariente $^{2} \cdot$ Bernard Bégaud $^{2} \cdot$ Hélène Verdoux $^{2,3}$. \\ Rosemary Dray-Spira ${ }^{1} \cdot$ Mahmoud Zureik ${ }^{1,4}$
}

Published online: 29 June 2018

(c) The Author(s) 2018

\begin{abstract}
Background Hepatotoxicity may be a concern when prescribing antidepressants. Nevertheless, this risk remains poorly understood for serotonin and noradrenaline reuptake inhibitors (SNRIs: venlafaxine, milnacipran, duloxetine) and 'other antidepressants' (mianserin, mirtazapine, tianeptine and agomelatine), particularly in comparison with selective serotonin reuptake inhibitors (SSRIs: fluoxetine, citalopram, paroxetine, sertraline, fluvoxamine, escitalopram), which are by far the most commonly prescribed antidepressants.

Objective We quantified the risk of serious liver injury associated with new use of SNRIs and 'other antidepressants' compared with SSRIs in real-life practice.

Methods Based on the French national health insurance database, this cohort study included 4,966,825 individuals aged 25 years and older with a first reimbursement of SSRIs, SNRIs or 'other antidepressants' between January 2010 and June 2015. We compared the risk of serious liver injury within the 6 months following antidepressant initiation according to antidepressant class, with SSRIs as the reference, using an inverse probability-of-treatment-weighted Cox proportional hazard model adjusted for demographic characteristics and risk factors of liver injury.

Results We identified 382 serious liver injuries overall (none for milnacipran initiators). Age and gender standardized incidence rates per 100,000 person-years were 19.2 for SSRIs, 22.2 for venlafaxine, 12.6 for duloxetine, 21.5 for mianserin, 32.8 for mirtazapine, 31.6 for tianeptine and 24.6 for agomelatine initiators. Initiation of antidepressants of interest versus SSRIs was not associated with an increased risk of serious liver injury [adjusted hazard ratios (95\% confidence interval): venlafaxine 1.17 (0.83-1.64), duloxetine 0.54 (0.28-1.02), mianserin 0.90 (0.58-1.41), mirtazapine 1.17 (0.67-2.02), tianeptine $1.35(0.82-2.23)$ and agomelatine $1.07(0.51-2.23)]$. This finding was confirmed by the results of an additional study using a case-time-control design.

Conclusion These results do not provide evidence of an increased risk of serious liver injury following initiation of SNRIs or 'other antidepressants' compared with SSRIs in real-life practice. This could reflect an inherent lack of difference in risk between the drug classes, or the fact that individuals with higher susceptibility to drug-induced liver injury are not prescribed drugs considered to be more hepatotoxic.
\end{abstract}

Electronic supplementary material The online version of this article (https://doi.org/10.1007/s40263-018-0537-1) contains supplementary material, which is available to authorized users.

Mahmoud Zureik

mahmoud.zureik@ansm.sante.fr

Extended author information available on the last page of the article 


\section{Key Points}

This cohort study, which included 4,966,825 antidepressant initiators identified in the French national health insurance database, did not identify any significant increased risk of serious liver injury associated with serotonin and noradrenaline reuptake inhibitors and 'other antidepressants' compared with selective serotonin reuptake inhibitors.

In real-life practice, the risk of serious liver injury does not seem to differ markedly across antidepressants proposed as first line. This could reflect an inherent lack of difference in risk between the drug classes, or the fact that individuals with higher susceptibility to druginduced liver injury are not prescribed drugs considered to be more hepatotoxic. hepatotoxicity mainly ensues from clinical trials and pharmacovigilance case reports [5]. Observational studies, tailored to evaluate such a risk in real-life conditions, are scarce, have limited sample sizes and mainly focused on duloxetine $[9,10]$.

The objective of the present study was to quantify the risk of serious liver injury associated with initiation of SNRIs (venlafaxine, milnacipran and duloxetine) and 'other antidepressants' (mianserin, mirtazapine, tianeptine and agomelatine) compared with initiation of SSRIs in real-life practice. Tricyclic antidepressants and monoamine oxidase inhibitors (MAOIs) were outside the scope of the study because of their particular safety profile, justifying their second-line use for the treatment of major depressive disorders.

\section{Methods}

\subsection{Data Sources}

A cohort study was conducted using the French national health insurance database [Système National d'Information Inter-Régimes de l'Assurance Maladie (SNIIRAM)]. Individuals were enrolled from January 1, 2010 to June 30, 2015. This health insurance database covers almost the entire French population (65.3 million inhabitants) with different affiliation schemes based on types of employment. For the present study, the data relating to beneficiaries of the main scheme (the general scheme covering approximately $77 \%$ of the French population) were used. The SNIIRAM comprehensively records the following with corresponding dates: outpatient drugs [Anatomical Therapeutic Classification (ATC) codes] and medical devices prescribed, reimbursed services and procedures, as well as demographic data such as age, sex, area of residence (postcode), complementary universal health insurance (free access to healthcare for people with an annual income $<50 \%$ of the poverty threshold) and date of death. The database does not stipulate the medical indication for each reimbursement. However, it includes information on severe and costly long-term disease (LTD) for which health expenses are fully reimbursed; these are coded according to the International Statistical Classification of Diseases and Related Health Problems, 10th Revision (ICD-10) and the date of LTD diagnosis is recorded. An anonymous unique identifier for each patient links SNIIRAM information to the national hospital discharge database [Programme de Médicalisation des Systèmes d'Information (PMSI)], which covers all hospitals and provides reasons for admission (in the format of the ICD-10) and surgical procedures. Previous observational studies using the SNIIRAM and the PMSI have been published elsewhere [11-17]. 


\subsection{Inclusion and Exclusion Criteria}

Individuals aged 25 years and older, affiliated to the general insurance scheme without changes in health plan during the previous 12 months and with a first reimbursement (i.e., no antidepressant reimbursed in the previous 6 months) of antidepressant (except tricyclic or MAOIs) between January 1, 2010 and June 30, 2015 were included in the study. Inclusion date corresponded to the first date meeting those conditions. In order to minimize the collection of non-drug-induced liver injury, individuals with a history of acute or chronic liver disease, pancreatitis, cancer, human immunodeficiency virus infection (HIV), alcohol use disorders identified based upon LTD or hospitalization diagnoses or reimbursements of specific drugs [Appendix Table 1, see electronic supplementary material (ESM)] were excluded due to pre-existing or possible liver conditions. Individuals with reimbursements for antidepressants of several groups (see exposure definition) within 15 days of inclusion were also excluded.

\subsection{Exposure Definition}

Eight groups of antidepressant initiators were identified depending on the molecule used at inclusion among those marketed in France during the study period: (1) SSRIs (ATC code, fluoxetine: N06AB03, citalopram: N06AB04, paroxetine: N06AB05, sertraline: N06AB06, fluvoxamine: N06AB08 or escitalopram: N06AB10), (2) venlafaxine (N06AX16), (3) milnacipran (N06AX17), (4) duloxetine (N06AX21), (5) mianserin (N06AX03), (6) mirtazapine (N06AX11), (7) tianeptine (N06AX14) and (8) agomelatine (N06AX22).

\subsection{Outcome Definition}

The outcome was defined as the occurrence of a serious liver injury possibly related to antidepressant exposure (i.e., acute form of serious liver injuries), resulting in hospitalization within the 6 months following antidepressant initiation. The outcome was identified by using the main or related diagnoses of incident hospital stays registered in the PMSI (ICD-10 codes: K71.0, K71.1, K71.2, K71.6, K71.8, K71.9, K72.0, K75.2, K75.9, K76.2, K76.7, Z94.4, Appendix Table 2, see ESM). A literature search was undertaken to identify ICD10 codes having a priori the best positive predictive value [18-21]. These codes were then validated by a senior pharmacologist (BB).

\subsection{Follow-Up}

Individuals were followed up for 6 months after antidepressant initiation or up to the first of the following events: serious liver injury, antidepressant discontinuation (follow-up pursued 3 months after discontinuation), switch to another antidepressant, cancer, HIV infection, viral hepatitis, change in health plan or death.

\subsection{Covariates}

The following covariates were identified at inclusion date: age categories, sex, quintiles of deprivation index of participant's area of residence (reflecting a major part of spatial socioeconomic heterogeneity) [22], complementary universal health insurance, prime-prescriber specialty (general practitioner, private practice psychiatrist or hospital practitioner) and inclusion year. Reimbursements for drugs potentially associated with hepatotoxicity (at least one reimbursement, Appendix Table 3, see ESM) [23, 24], and immunosuppressant drugs (at least two reimbursements, ATC code L04A) were considered respectively in the 6 and 12 months preceding inclusion (including the date of antidepressant initiation).

The following comorbidities were considered on the basis of relevant reimbursed medicines (at least two reimbursements, ATC codes), hospital discharge or LTD reports (ICD10 diagnosis codes): psychiatric history (based upon psychotropic drug reimbursement or psychiatric disease diagnosis), diabetes mellitus (based upon antidiabetic drug reimbursement or diagnosis related to diabetes and its complications), cardiac failure (based upon related diagnosis), chronic renal failure (based upon related diagnosis), measurable morbid obesity (based upon related diagnosis) in the 12 months preceding inclusion (including the date of antidepressant initiation), measurable history of substance abuse (based upon opiate substitution reimbursement or diagnosis related to substance abuse) and smoking habits (based upon nicotine replacement therapy reimbursement or diagnosis related to tobacco use) in the 36 months preceding inclusion (including the date of antidepressant initiation). Detailed ATC and ICD-10 codes used to identify comorbidities are provided in Appendix Table 4 (see ESM).

Individuals with reimbursement for aminotransferase testing in the month preceding or following inclusion were identified as well as those with initiation of drugs potentially associated with hepatotoxicity, additional antidepressants or aminotransferase testing during the follow-up.

\subsection{Statistical Analysis}

Antidepressant initiators were described according to the distribution of covariates previously mentioned. Sex- and age-standardized incidences of serious liver injury were computed for each group of antidepressant initiators based upon sex and age distribution of SSRI initiators. A univariate Cox proportional hazard model with initiation of antidepressant as the main explanatory variable was first 
used to compare the risk of serious liver injury according to initiation of venlafaxine, milnacipran, duloxetine, mianserin, mirtazapine, tianeptine and agomelatine versus SSRIs. To control for confounding, an inverse probabilityof-treatment-weighted Cox proportional hazard model for incident liver injuries was then used. Weights were derived from the reciprocal of the propensity scores containing the following characteristics assessed at inclusion: age categories, sex, year of inclusion, deprivation quintiles, complementary universal health insurance, diabetes, heart failure, chronic renal failure, reimbursement for drugs potentially associated with hepatotoxicity, reimbursement for immunosuppressant drugs, measurable history of substance abuse or smoking habits, morbid obesity and aminotransferase testing. To reduce instability induced by large weights, the stabilized weights were computed and then were truncated at the first and 99th percentiles [25]. The standardized differences method for assessing balance in observed baseline covariates between the SSRIs and each antidepressant of interest was applied to compare prevalence of covariates in the stabilized weighted sample. Imbalances below $10 \%$ were considered as negligible [26]. An additional adjustment was made on variables strongly linked with exposure (i.e., psychiatric history, prime-prescriber specialty) [27], time-varying covariates (i.e., initiation of drugs potentially associated with hepatotoxicity or other antidepressants, aminotransferase testing during follow-up), and age categories.

\subsection{Sensitivity Analysis}

In a first sensitivity analysis, the code K72.9 'hepatic failure unspecified' was added to the definition of events. Although poorly specific, this code was considered to be compatible with a potential DILI. In a second sensitivity analysis, the follow-up duration was extended to 12 months after antidepressant initiation. In a third sensitivity analysis, the sample was stratified according to the presence or not of aminotransferase testing at inclusion to assess the influence of possible bias ensuing from differential care related to the forthcoming outcome. Finally, a stratification on the number of prescriptions of antidepressant reimbursed during the follow-up (one versus more than one, one reimbursement corresponding to a maximum 30-day supply) was undertaken to control for the bias possibly caused by individuals with a single reimbursement, since they were likely to be non-compliant and therefore not exposed.

\subsection{Secondary Approach: Study Using a Case-Time-Control Design}

A study using a case-time-control design was undertaken as a secondary approach to challenge the conclusions of the main cohort approach and to better control for residual unmeasured confounding; that is, related to variables not directly recorded in automated databases and considered as unchanged over the study period (e.g., disorders related to alcohol abuse, smoking, morbid obesity etc.). One analysis was performed for each antidepressant of interest compared with SSRIs.

In each analysis, incident cases of serious liver injury occurring between September 1, 2011 and June 30, 2015 were identified using the same criteria as for the main cohort approach. Exposure to antidepressants of interest and SSRIs was measured both during a 'risk' and a 'reference' period corresponding respectively to the 6 months preceding the liver-related event date and to the period from the 9th to the 15 th month before this date, a 3-month wash-out period separating these two time windows.

A conditional logistic regression was used to evaluate whether, comparatively to SSRIs, exposure to the antidepressant of interest was more prevalent during the 'risk' period compared with the 'reference' period. Antidepressant users considered for analyses were individuals with reimbursements for both (1) SSRIs in the absence of antidepressant of interest during one of the two periods and (2) antidepressant of interest in the absence of SSRIs during the other period (Fig. 1). Odds ratios (ORs), crude and adjusted on covariates prone to vary across the periods (i.e., other antidepressants and drugs potentially associated with hepatotoxicity), were computed.

The particularity of the case-time-control design lies in introducing a specific control group to control for temporal biases; that is, when the probability of exposure could be affected by temporal trends independent of the event of interest (e.g., launch of a new drug, introduction of safety warnings, seasonality, etc.). Therefore, for each case, 20 controls of the same sex and age ( \pm 1 year) were randomly sampled from the 'non-case' population. Exposure was measured as for the matched case, and, taking into account exposure temporal trends, ORs were derived from the coefficient of the interaction between exposure and case or control status introduced in the conditional logistic regressions models [28].

All statistical tests were two-tailed with an $\alpha$-risk of 5\%. Statistical analyses were performed using SAS Enterprise Guide software, version 7.1 (SAS Institute Inc.).

\section{Results}

\subsection{Inclusion and Follow-Up Characteristics of Antidepressant Initiators}

The study included 4,966,825 antidepressant initiators (Fig. 2). Their characteristics at inclusion are described in Table 1. Women accounted for $68 \%$ of the sample. Mean age was 

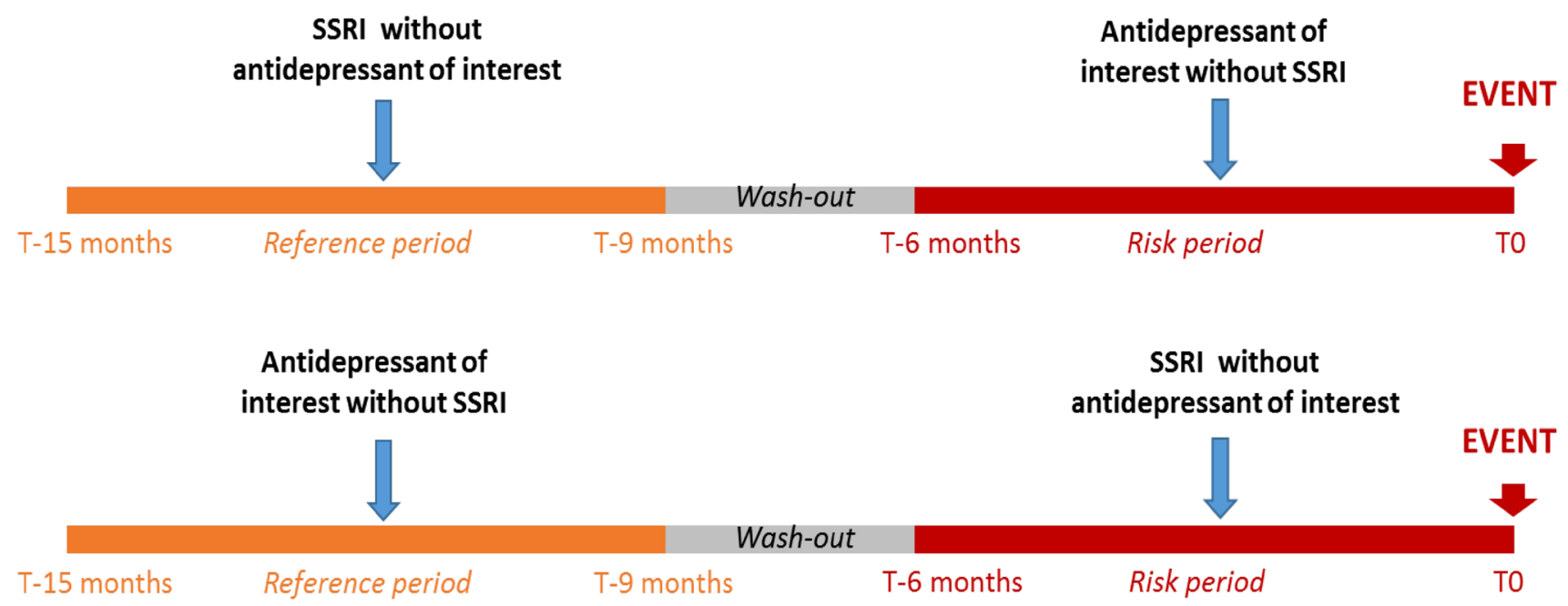

Fig. 1 Exposure to antidepressants of interest and selective serotonin reuptake inhibitors (SSRIs) for cases and controls included in the casetime-control design according to study period

52 years, agomelatine initiators were younger and mianserin, mirtazapine and tianeptine initiators were older. Around 8\% had complementary universal health insurance. Most of the time the prime prescriber was a general practitioner. For milnacipran, mirtazapine and agomelatine initiators, the proportion of prescriptions initiated by a private practice psychiatrist ranged from 8 to $15 \%$ versus $<7 \%$ for other groups. Aminotransferase testing at inclusion was in place for $35 \%$ of agomelatine initiators versus around $20 \%$ in the other groups. A history of psychiatric disorders was more frequently found in all groups than among SSRI initiators and was more prevalent in the milnacipran, mianserin, mirtazapine and agomelatine groups. Diabetes was more frequent in duloxetine, mianserin, mirtazapine and tianeptine initiators. Heart failure and chronic renal failure were more often found in mianserin, mirtazapine and tianeptine initiators. Mean follow-up was around 4 months. For $40-50 \%$ of initiators, only one reimbursement of antidepressant was retrieved during the follow-up (Appendix Table 5, see ESM).

The mean (SD) estimated stabilized weight was 1.00 (0.31), ranging from 0.35 to 2.18 . The standard difference in the unweighted model exceeded $10 \%$ for $24.9 \%$ of the sample. After weighting by the inverse probability of treatment (and stabilization and truncation of the weights), prevalence of qualitative categorical variables was similar between each antidepressant of interest and SSRIs (Appendix Table 6, see ESM).

\subsection{Main Approach: Association Between Antidepressant of Interest and Serious Liver Injury}

A total of 382 serious liver injuries were identified (258 for SSRIs, 36 for venlafaxine, 12 for duloxetine, 29 for mianserin, 15 for mirtazapine, 24 for tianeptine, 8 for agomelatine, see description in Appendix Table 2, ESM) and occurred with a mean delay of 2 months after treatment initiation. No event was identified for milnacipran. Sex- and age-standardized incidence rates per 100,000 person-years were 19.2 for SSRIs, 22.2 for venlafaxine, 12.6 for duloxetine, 21.5 for mianserin, 32.8 for mirtazapine, 31.6 for tianeptine and 24.6 for agomelatine. Initiation of antidepressants of interest was not associated with a significant increased risk of serious liver injury when compared with SSRIs; adjusted hazard ratios $(95 \% \mathrm{CI}) 1.17(0.83-1.64), p=0.36$ for venlafaxine; $0.54(0.28-1.02), p=0.06$ for duloxetine; $0.90(0.58-1.41), p=0.64$ for mianserin; $1.17(0.67-2.02)$, $p=0.56$ for mirtazapine; $1.35(0.82-2.23), p=0.24$ for tianeptine and $1.07(0.51-2.23), p=0.86$ for agomelatine. Results are summarized in Table 2.

\subsection{Sensitivity Analysis}

Adding the code K72.9 'hepatic failure unspecified' to the main definition of the outcome (37 incident events added) or 


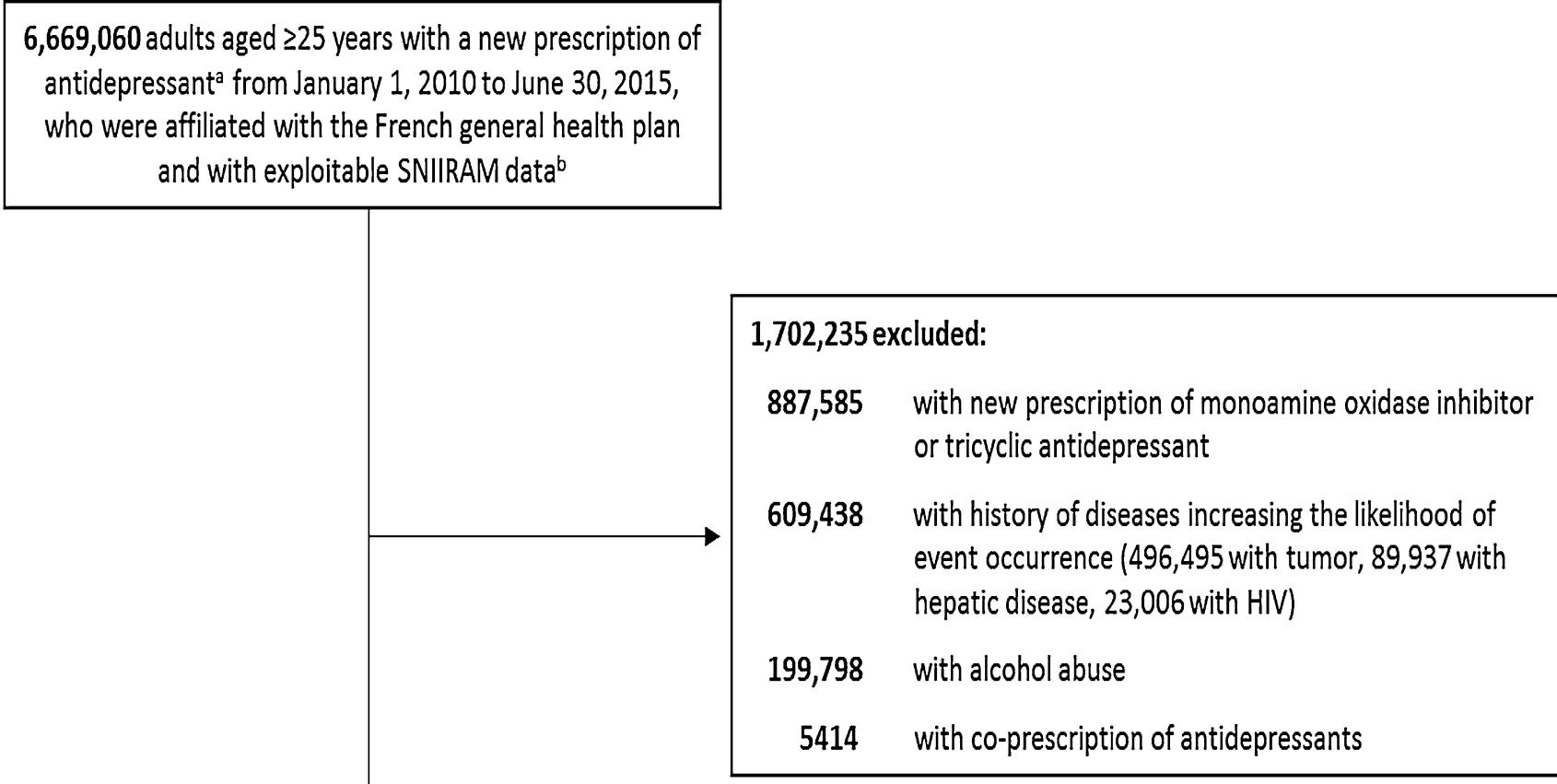

4,966,825 included in the study population:

$3,543,559$ SSRl initiators

436,155 venlafaxine initiators

37,577 milnacipran initiators

247,250 duloxetine initiators

293,484 mianserin initiators

128,593 mirtazapine initiators

181,289 tianeptine initiators

98,918 agomelatine initiators

a No prescription of antidepressant in the previous 6 months.

b 1,072,879 individuals with change in health plan, 351,139 twins and 440 individuals with cancelled antidepressant prescription were not included in the study population.

Fig. 2 Diagram of inclusion. HIV human immunodeficiency virus, SNIIRAM Système National d'Information Inter-Régimes de l'Assurance Maladie, SSRIs selective serotonin reuptake inhibitors

extending follow-up from 6 to 12 months (116 events added) did not significantly alter the results (Appendix Tables 7 and 8, see ESM). Conclusions remained unchanged in analyses stratified on aminotransferase testing at inclusion and on the number of antidepressant prescriptions during the follow-up (Appendix Tables 9 and 10, see ESM).

\subsection{Secondary Approach: Study Using a Case-Time-Control Design}

In this approach, when comparing exposure during 'risk' and 'reference' periods and using SSRIs as controls, the adjusted ORs (95\% CI) for antidepressants of interest were $0.94(0.51-1.72), p=0.84$ for venlafaxine; 0.86 


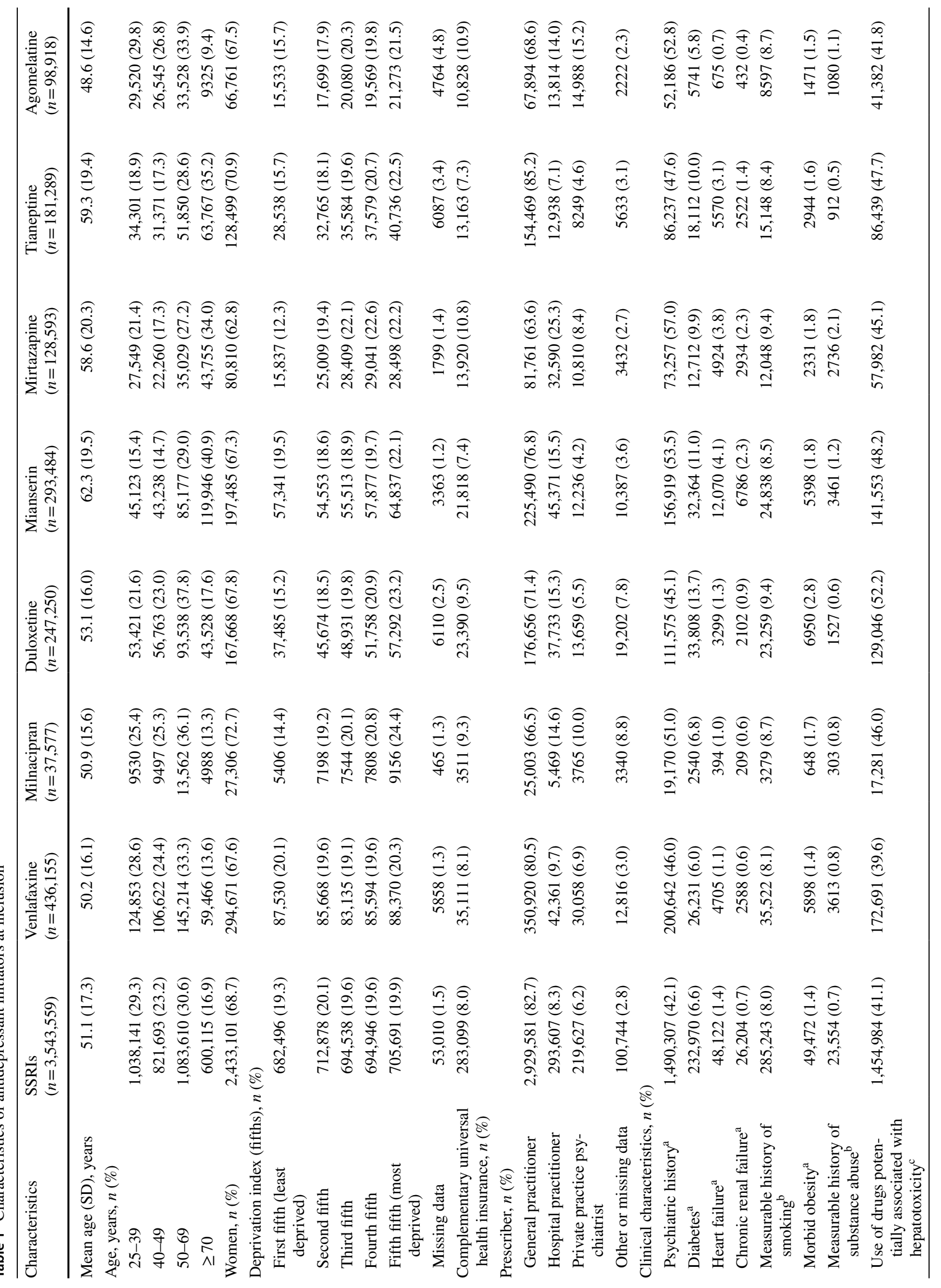




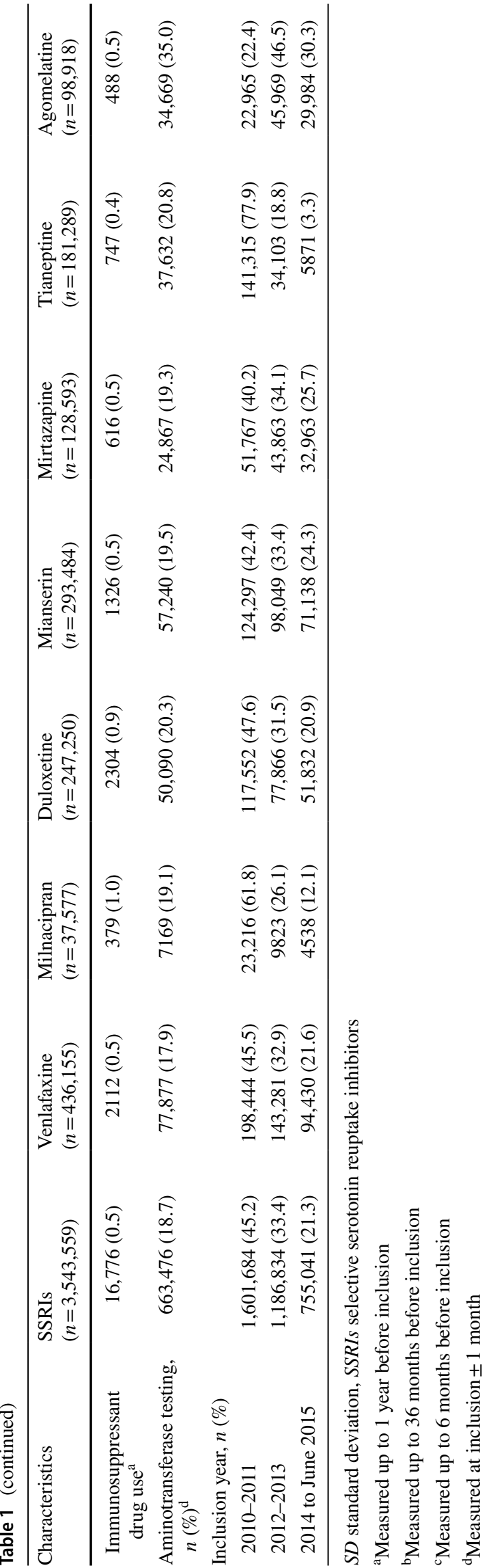

(0.12-5.98), $p=0.88$ for milnacipran; $1.15(0.53-2.53)$, $p=0.72$ for duloxetine; $0.91(0.42-1.97), p=0.82$ for mianserin; 1.13 (0.43-3.00), $p=0.80$ for mirtazapine; 1.06 (0.40-2.82), $p=0.91$ for tianeptine and $0.87(0.12-6.34)$, $p=0.89$ for agomelatine (Table 3 ).

\section{Discussion}

This large prospective observational study conducted in an extensive and representative sample of the French population did not identify any increased risk of serious liver injury associated with the use of SNRIs (venlafaxine, milnacipran and duloxetine) or 'other antidepressants' (mianserin, mirtazapine, tianeptine and agomelatine) when compared with SSRIs in real-life practice. This conclusion was supported by the results of a case-time-control analysis conducted as a secondary approach.

Most of the data concerning antidepressant hepatotoxicity ensue from pharmacovigilance and published case reports. Some observational studies of limited sample size have provided information about the hepatotoxicity of duloxetine and agomelatine as they were subjected to risk management plans. Two observational studies specifically designed to evaluate duloxetine hepatotoxicity suggested a higher incidence of liver injuries other than hepatic-related death or liver failure with this molecule compared with several other antidepressants, but the conclusions were hampered by insufficient statistical power $[9,10]$. Interestingly, our main analysis suggests a trend toward a lower hepatotoxicity of duloxetine when compared with SSRIs but this result was both non-significant and not confirmed by the secondary case-time-control approach. Two observational studies conducted to assess agomelatine safety did not highlight any serious case of hepatotoxicity [29,30], even though they did not specifically focus on hepatotoxicity and were not comparative. Similarly, our study does not support the existence of a higher risk of serious hepatotoxicity for agomelatine initiators when compared with SSRIs in real conditions of use.

There is clearly a lack of observational studies concerning the potential hepatotoxicity of non-SSRI antidepressants. Possible hepatotoxicity of non-SSRI antidepressants was deduced from a rise in aminotransferase over the normal threshold or rarely by the occurrence of a clinical liver injury during pre-marketing clinical trials or post-approval studies $[6,31,32]$ or in published case reports [33-39].

Several interpretations can be put forward to explain the absence of a significant difference in the risk of serious hepatotoxicity between the antidepressants of interest and SSRIs in real-life settings. The first and most obvious is that the antidepressants suspected of conveying the highest risks of hepatotoxicity are not prescribed to individuals believed to present higher susceptibility to DILI (e.g., 
Table 2 Association between initiation of antidepressants of interest versus selective serotonin reuptake inhibitors (SSRIs) and hospitalization due to serious liver injury (main definition of event, follow-up 6 months)

\begin{tabular}{|c|c|c|c|c|c|}
\hline \multirow[t]{2}{*}{ Antidepressant } & \multirow{2}{*}{$\begin{array}{l}\text { Initiators } \\
(n=4,966,825)\end{array}$} & \multirow[t]{2}{*}{ Events $(n=382)$} & \multirow{2}{*}{$\begin{array}{l}\text { Event incidence (per 100,000 } \\
\text { person-years) }^{\mathrm{a}}\end{array}$} & \multicolumn{2}{|c|}{ Hazard ratio $(95 \% \mathrm{CI})$} \\
\hline & & & & Crude & Adjusted $^{\mathrm{b}, \mathrm{c}}$ \\
\hline SSRIs & $3,543,559$ & 258 & 19.2 & 1.00 [Reference] & 1.00 [Reference] \\
\hline Venlafaxine & 436,155 & 36 & 22.2 & $1.15(0.81-1.63)$ & $1.17(0.83-1.64)$ \\
\hline Milnacipran & 37,577 & 0 & 0.0 & & \\
\hline Duloxetine & 247,250 & 12 & 12.6 & $0.70(0.39-1.24)$ & $0.54(0.28-1.02)$ \\
\hline Mianserin & 293,484 & 29 & 21.5 & $1.43(0.97-2.10)$ & $0.90(0.58-1.41)$ \\
\hline Mirtazapine & 128,593 & 15 & 32.8 & $1.65(0.98-2.77)$ & $1.17(0.67-2.02)$ \\
\hline Tianeptine & 181,289 & 24 & 31.6 & $1.93(1.27-2.94)$ & $1.35(0.82-2.23)$ \\
\hline Agomelatine & 98,918 & 8 & 24.6 & $1.18(0.58-2.38)$ & $1.07(0.51-2.23)$ \\
\hline
\end{tabular}

CI confidence interval, SSRIs selective serotonin reuptake inhibitors

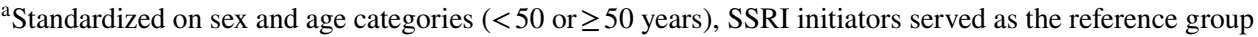

${ }^{\mathrm{b}}$ Inverse probability of treatment weighting considering the following covariates: inclusion year, sex, age, deprivation index and complementary universal health insurance at inclusion; diabetes, heart failure, chronic renal failure, measurable history of smoking, morbid obesity and measurable history of substance abuse up to $12-36$ months before inclusion; reimbursements for drugs potentially associated with hepatotoxicity and immunosuppressant drugs up to 6-12 months before inclusion; aminotransferase testing at inclusion \pm 1 month

${ }^{\mathrm{c}}$ Additional adjustment on age categories, prescriber specialty at inclusion; psychiatric history in 12 months before inclusion; drugs potentially associated with hepatotoxicity, other antidepressants and aminotransferase testing reimbursed during follow-up

Table 3 Odds ratios for hospitalization due to serious liver injury associated with antidepressant of interest versus selective serotonin reuptake inhibitors (SSRIs) in each case-time-control study performed by antidepressant of interest

\begin{tabular}{|c|c|c|c|c|}
\hline \multirow{2}{*}{$\begin{array}{l}\text { Antidepressant of interest in each case- } \\
\text { time-control study }\end{array}$} & \multicolumn{2}{|l|}{ Cases } & \multicolumn{2}{|c|}{ Odds ratio $(95 \% \mathrm{CI})$} \\
\hline & Reference period $(n)^{\mathrm{b}}$ & Risk period $(n)^{\mathrm{c}}$ & Crude & Adjusted $^{\mathrm{d}}$ \\
\hline Venlafaxine & 21 & 28 & $1.00(0.56-1.81)$ & $0.94(0.51-1.72)$ \\
\hline Milnacipran & 5 & 2 & $0.43(0.08-2.36)$ & $0.86(0.12-5.98)$ \\
\hline Duloxetine & 13 & 18 & $1.09(0.52-2.30)$ & $1.15(0.53-2.53)$ \\
\hline Mianserin & 16 & 21 & $1.07(0.54-2.11)$ & $0.91(0.42-1.97)$ \\
\hline Mirtazapine & 11 & 13 & $0.93(0.40-2.18)$ & $1.13(0.43-3.00)$ \\
\hline Tianeptine & 17 & 7 & $0.94(0.37-2.36)$ & $1.06(0.40-2.82)$ \\
\hline Agomelatine & 5 & 4 & $0.69(0.18-2.69)$ & $0.87(0.12-6.34)$ \\
\hline
\end{tabular}

CI confidence interval, SSRIs selective serotonin reuptake inhibitors

${ }^{a}$ Eligible profiles in each case-time-control study performed by antidepressant of interest: individuals with reimbursements for both (1) antidepressant of interest in the absence of SSRIs during one of the two periods (i.e., risk or reference) and (2) SSRIs in the absence of antidepressant of interest during the other period

${ }^{b}$ Number of cases with reimbursements for both (1) antidepressant of interest in the absence of SSRIs during the reference period and (2) SSRIs in the absence of antidepressant of interest during the risk period

${ }^{c}$ Number of cases with reimbursements for both (1) antidepressant of interest in the absence of SSRIs during the risk period and (2) SSRIs in the absence of antidepressant of interest during the reference period

${ }^{\mathrm{d}}$ Adjusted for variations in drugs potentially associated with hepatotoxicity and other antidepressant reimbursement between reference and risk periods

elderly, individuals with diabetes, heart failure, chronic renal failure etc.). This is particularly the case for products involved in risk management plans such as agomelatine and duloxetine, thanks to the information about their hepatotoxicity provided to prescribers. Second, for particular risk situations or if evocative symptoms are identified, it is likely that aminotransferases would be monitored and the treatment stopped before the occurrence of a serious outcome. However, this hypothesis is not supported by the fact that stratifying on liver monitoring at inclusion did not significantly alter the results. Finally, it is worth recalling that this study was designed to assess the risk of serious hepatotoxicity, so it did not consider infra-clinical or non-serious events, as 
was the case for previous observational studies conducted for duloxetine $[9,10]$.

To our knowledge, the present study is the first to compare the risk of the hepatotoxicity of antidepressants both in real life and on the basis of such a large (5 million treatment initiators) representative nationwide sample. Its sample size warranted identifying rare outcomes such as serious, including fatal, DILIs. The study also utilized the large amount of information provided by the SNIIRAM database to control for the main potential confounders by means of propensity scores, thereby providing more reliable estimates compared with classic multivariate models in the event of rare outcomes [40]. It is also likely that using an active comparator minimized the influence of measured and unmeasured differences in patient characteristics linked with both antidepressant use and the risk of hepatotoxicity [41]. Moreover, the conclusions remained unchanged in several sensitivity analyses testing the effect of a broader event definition, longer follow-up (i.e., 12 months, even if liver-related events generally appear in the 6 months after drug initiation), liver monitoring or excluding patients with a single reimbursement of antidepressant. Finally, the case-time-control design used as a secondary approach to better control for potential unmeasured invariant confounders confirmed the conclusions.

This study also has several limitations. The data were fully anonymized so accessing the original medical files to complement and ascertain clinical information on cases of liver injury was not possible. Therefore, the predictive positive value of the ICD-10 codes used to identify these cases without clinical validation could be debated [19] and the incidence rates obtained may not reflect true incidence rates of DILI. However, given the type of hepatotoxicity considered-serious cases leading to hospitalization or death-and the fact that making the definition broader did not alter the results, it is likely that this bias, if any, did not have a marked influence on the results. Moreover, it would apply the same way in each comparison group and would not have masked a significant excess risk of hepatotoxicity in users of the antidepressants of interest compared with SSRIs.

\section{Conclusion}

This study conducted on almost 5 million antidepressant initiators did not provide any evidence of an increased risk of serious liver injury associated with SNRIs (venlafaxine, milnacipran and duloxetine) or 'other antidepressants' (mianserin, mirtazapine, tianeptine and agomelatine) when compared with SSRIs in real-life settings. These findings could reflect an inherent lack of difference in risk between the drug classes, or the fact that individuals with higher susceptibility to drug-induced liver injury are not prescribed drugs considered to be more hepatotoxic.
Author Contributions $\mathrm{CC}, \mathrm{SB}, \mathrm{RD}$ and $\mathrm{MZ}$ contributed to the conception and design of the study. CC, SB and TL did the statistical analyses. All authors contributed to interpretation of the results. SB drafted the manuscript. All authors critically revised the manuscript for important intellectual content. SB and CC have full access to all the data in the study and take responsibility for the integrity of the data and the accuracy of the data analysis. RD and MZ supervised the study. All the authors made a significant contribution to the research and the development of the manuscript and approved the final version for publication. $\mathrm{SB}$ and $\mathrm{CC}$ are guarantors.

\section{Compliance with Ethical Standards}

Conflict of interest S Billioti de Gage, C. Collin, T. Le-Tri, A. Pariente, B. Bégaud, H. Verdoux, R. Dray-Spira and M. Zureik have no conflicts of interest to disclose.

Funding The open access fee was funded by the French National Agency for Medicines and Health Products Safety (ANSM). The authors have no financial support to disclose. All authors declare no support from any organization for the submitted work and no financial relationships with any organizations that might have an interest in the submitted work.

Open Access This article is distributed under the terms of the Creative Commons Attribution-NonCommercial 4.0 International License (http://creativecommons.org/licenses/by-nc/4.0/), which permits any noncommercial use, distribution, and reproduction in any medium, provided you give appropriate credit to the original author(s) and the source, provide a link to the Creative Commons license, and indicate if changes were made.

\section{References}

1. Ostapowicz G, Fontana RJ, Schiødt FV, Larson A, Davern TJ, Han $\mathrm{SHB}$, et al. Results of a prospective study of acute liver failure at 17 tertiary care centers in the United States. Ann Intern Med. 2002;137:947-54.

2. Navarro VJ, Senior JR. Drug-related hepatotoxicity. N Engl J Med. 2006;354:731-9.

3. Sgro C, Clinard F, Ouazir K, Chanay H, Allard C, Guilleminet $\mathrm{C}$, et al. Incidence of drug-induced hepatic injuries: a French population-based study. Hepatology. 2002;36:451-5.

4. Björnsson E, Bergmann O, Björnsson H, Kvaran R, Olafsson $\mathrm{S}$. Incidence, presentation, and outcomes in patients with druginduced liver injury in the general population of Iceland. Gastroenterology. 2013;144:1419-25.

5. Voican CS, Corruble E, Naveau S, Perlemuter G. Antidepressantinduced liver injury: a review for clinicians. Am J Psychiatry. 2014;171:404-15.

6. Friedrich M-E, Akimova E, Huf W, Konstantinidis A, Papageorgiou $\mathrm{K}$, Winkler $\mathrm{D}$, et al. Drug-induced liver injury during antidepressant treatment: results of AMSP, a drug surveillance program. Int J Neuropsychopharmacol. 2016;19(4):1-9.

7. Wernicke J, Pangallo B, Wang F, Murray I, Henck JW, Knadler MP, et al. Hepatic effects of duloxetine-I: non-clinical and clinical trial data. Curr Drug Saf. 2008;3:132-42.

8. Freiesleben SD, Furczyk K. A systematic review of agomelatineinduced liver injury. J Mol Psychiatry. 2015;3:4.

9. Lin ND, Norman H, Regev A, Perahia DG, Li H, Chang CL, et al. Hepatic outcomes among adults taking duloxetine: a retrospective 
cohort study in a US health care claims database. BMC Gastroenterol. 2015;15:134.

10. Xue F, Strombom I, Turnbull B, Zhu S, Seeger JD. Duloxetine for depression and the incidence of hepatic events in adults. J Clin Psychopharmacol. 2011;31:517-22.

11. Lemaitre M, Kirchgesner J, Rudnichi A, Carrat F, Zureik M, Carbonnel $\mathrm{F}$, et al. Association between use of thiopurines or tumor necrosis factor antagonists alone or in combination and risk of lymphoma in patients with inflammatory bowel disease. JAMA. 2017;318:1679-86.

12. Weill A, Dalichampt M, Raguideau F, Ricordeau $\mathrm{P}$, Blotière $\mathrm{P}-\mathrm{O}$, Rudant J, et al. Low dose oestrogen combined oral contraception and risk of pulmonary embolism, stroke, and myocardial infarction in five million French women: cohort study. BMJ. 2016;353:i2002.

13. Raguideau F, Lemaitre M, Dray-Spira R, Zureik M. Association between oral fluoroquinolone use and retinal detachment. JAMA Ophthalmol. 2016;134:415-21.

14. Bouillon K, Bertrand M, Boudali L, Ducimetière P, Dray-Spira $\mathrm{R}$, Zureik M. Short-term risk of bleeding during heparin bridging at initiation of vitamin $\mathrm{K}$ antagonist therapy in more than 90,000 patients with nonvalvular atrial fibrillation managed in outpatient care. J Am Heart Assoc. 2016;5(11):e004065.

15. Colas S, Collin C, Piriou P, Zureik M. Association between total hip replacement characteristics and 3-year prosthetic survivorship: a population-based study. JAMA Surg. 2015;150:979-88.

16. Bouillon K, Bertrand M, Maura G, Blotière P-O, Ricordeau P, Zureik M. Risk of bleeding and arterial thromboembolism in patients with non-valvular atrial fibrillation either maintained on a vitamin $\mathrm{K}$ antagonist or switched to a non-vitamin K-antagonist oral anticoagulant: a retrospective, matched-cohort study. Lancet Haematol. 2015;2:e150-9.

17. Maura G, Blotière P-O, Bouillon $\mathrm{K}$, Billionnet $\mathrm{C}$, Ricordeau $\mathrm{P}$, Alla F, et al. Comparison of the short-term risk of bleeding and arterial thromboembolic events in nonvalvular atrial fibrillation patients newly treated with dabigatran or rivaroxaban versus vitamin $\mathrm{K}$ antagonists: a French nationwide propensity-matched cohort study. Circulation. 2015;132:1252-60.

18. Carson JL, Strom BL, Duff A, Gupta A, Shaw M, Das K. The feasibility of studying drug-induced acute hepatitis with use of Medicaid data. Clin Pharmacol Ther. 1992;52:214-9.

19. Lo Re V III, Haynes K, Goldberg D, Forde KA, Carbonari DM, Leidl KBF, et al. Validity of diagnostic codes to identify cases of severe acute liver injury in the US Food and Drug Administration's Mini-Sentinel Distributed Database. Pharmacoepidemiol Drug Saf. 2013;22:861-72.

20. Lo Re V III, Carbonari DM, Forde KA, Goldberg D, Lewis JD, Haynes K, et al. Validity of diagnostic codes and laboratory tests of liver dysfunction to identify acute liver failure events. Pharmacoepidemiol Drug Saf. 2015;24:676-83.

21. Udo R, Maitland-van der Zee AH, Egberts TCG, den Breeijen JH, Leufkens HGM, van Solinge WW, et al. Validity of diagnostic codes and laboratory measurements to identify patients with idiopathic acute liver injury in a hospital database. Pharmacoepidemiol Drug Saf. 2015;25(Suppl-1):21-8.

22. Rey G, Jougla E, Fouillet A, Hémon D. Ecological association between a deprivation index and mortality in France over the period 1997-2001: variations with spatial scale, degree of urbanicity, age, gender and cause of death. BMC Public Health. 2009;9:33

23. Bjornsson ES. Hepatotoxicity by drugs: the most common implicated agents. Int J Mol Sci. 2016;17(2):224.
24. Suzuki A, Andrade RJ, Bjornsson E, Lucena MI, Lee WM, Yuen NA, et al. Drugs associated with hepatotoxicity and their reporting frequency of liver adverse events in VigiBase: unified list based on international collaborative work. Drug Saf. 2010;33:503-22.

25. Austin PC. The use of propensity score methods with survival or time-to-event outcomes: reporting measures of effect similar to those used in randomized experiments. Stat Med. 2014;33:1242-58.

26. Austin PC, Stuart EA. Moving towards best practice when using inverse probability of treatment weighting (IPTW) using the propensity score to estimate causal treatment effects in observational studies. Stat Med. 2015;34:3661-79.

27. Brookhart MA, Schneeweiss S, Rothman KJ, Glynn RJ, Avorn J, Stürmer T. Variable selection for propensity score models. Am J Epidemiol. 2006;163:1149-56.

28. Suissa S. The case-time-control design. Epidemiology. 1995;6:248-53.

29. Sparshatt A, McAllister Williams RH, Baldwin DS, Haddad PM, Bazire S, Weston E, et al. A naturalistic evaluation and audit database of agomelatine: clinical outcome at 12 weeks. Acta Psychiatr Scand. 2013;128:203-11.

30. Laux G, VIVALDI Study. Group. The antidepressant agomelatine in daily practice: results of the non-interventional study VIVALDI. Pharmacopsychiatry. 2012;45:284-91.

31. Gahr M, Zeiss R, Lang D, Connemann BJ, Schonfeldt-Lecuona C. Hepatotoxicity associated with agomelatine and other antidepressants: disproportionality analysis using pooled pharmacovigilance data from the Uppsala Monitoring Centre. J Clin Pharmacol. 2015;55:768-73.

32. Gahr M, Zeiss R, Lang D, Connemann BJ, Hiemke C, SchonfeldtLecuona $\mathrm{C}$. Drug-induced liver injury associated with antidepressive psychopharmacotherapy: an explorative assessment based on quantitative signal detection using different MedDRA terms. J Clin Pharmacol. 2016;56(6):769-78.

33. Macaigne G, Auriault M-L, Chayette C, Cheiab S, Deplus R. Tianeptine induced acute hepatitis. Gastroenterologie clinique et biologique. 2003;27:348-9.

34. Le Bricquir Y, Larrey D, Blanc P, Pageaux GP, Michel H. Tianeptine - an instance of drug-induced hepatotoxicity predicted by prospective experimental studies. J Hepatol. 1994;21:771-3.

35. Fromenty B, Pessayre D. Inhibition of mitochondrial betaoxidation as a mechanism of hepatotoxicity. Pharmacol Ther. 1995;67:101-54.

36. Stadlmann S, Portmann S, Tschopp S, Terracciano LM. Venlafaxine-induced cholestatic hepatitis: case report and review of literature. Am J Surg Pathol. 2012;36:1724-8.

37. Phillips BB, Digmann RR, Beck MG. Hepatitis associated with low-dose venlafaxine for postmenopausal vasomotor symptoms. Ann Pharmacother. 2006;40:323-7.

38. Hui C-K, Yuen M-F, Wong W-M, Lam S-K, Lai C-L. Mirtazapine-induced hepatotoxicity. J Clin Gastroenterol. 2002;35:270-1.

39. Kang S-G, Yoon B-M, Park Y-M. Mirtazapine-induced hepatocellular-type liver injury. Ann Pharmacother. 2011;45:825-6.

40. Glynn RJ, Schneeweiss S, Stürmer T. Indications for propensity scores and review of their use in pharmacoepidemiology. Basic Clin Pharmacol Toxicol. 2006;98:253-9.

41. Lund JL, Richardson DB, Stürmer T. The active comparator, new user study design in pharmacoepidemiology: historical foundations and contemporary application. Curr Epidemiol Rep. $2015 ; 2: 221-8$. 


\section{Affiliations}

Sophie Billioti de Gage ${ }^{1,2}$ (1) $\cdot$ Cédric Collin ${ }^{1} \cdot$ Thien Le-Tri $^{1} \cdot$ Antoine Pariente $^{2} \cdot$ Bernard Bégaud $^{2} \cdot$ Hélène Verdoux $^{2,3}$. Rosemary Dray-Spira ${ }^{1} \cdot$ Mahmoud Zureik ${ }^{1,4}$

1 Department of Epidemiology of Health Products, French National Agency for Medicines and Health Products Safety (ANSM), 143-147 Blvd Anatole France, 93285 Saint-Denis, France

2 University of Bordeaux, Inserm, Bordeaux Population Health Research Center, Team Pharmacoepidemiology, UMR 1219, 33000 Bordeaux, France
3 Pôle UNIVA (UNIVersitaire de psychiatrie Adulte), Centre Hospitalier Charles Perrens, 33000 Bordeaux, France

4 University of Versailles Saint-Quentin-en-Yvelines, 78180 Montigny-Le-Bretonneux, France 